\title{
Mass and moment of inertia constraints on the lunar crustal thickness: Relations between crustal density, mantle density, and the reference radius of the crust-mantle boundary
}

\author{
Hajime Hikida $^{1}$ and Hitoshi Mizutani ${ }^{2}$ \\ ${ }^{1}$ Institute de Physique du Globe de Paris, 4 Avenue de Neptune, Saint-Maur 94107, France \\ ${ }^{2}$ Japan Aerospace Exploration Agency, 3-1-1 Yoshinodai, Sagamihara, Kanagawa 229-8510, Japan \\ (Received March 25, 2005; Revised July 21, 2005; Accepted July 23, 2005)
}

\begin{abstract}
The gravity inversion for the lunar crustal thickness needs three parameters: the crustal density, mantle density, and the reference radius of the crust-mantle boundary. The assumption of crust and mantle densities is found to significantly affect on the resultant crustal thickness variations. Moreover, the recent seismic analyses suggest the possibility of thinner crustal thickness around the Apollo 12 site than that proposed in the Apollo-era, on which the previous gravity inversions relied upon. Therefore the validity of the assumption used in previous gravity-inversion studies must be re-examined in detail. By using a simple three-layered mass model of the Moon including a uniform crust, mantle, and core, we determine possible combinations of the three parameters, satisfying the mass and moment of inertia constraints. The results show that the set of the parameters used in Neumann et al. (1996) can be consistent with mass and moment of inertia constraints, while those in Wieczorek and Phillips (1998) does not satisfy the constraints.
\end{abstract}

Key words: Gravity inversion, crustal thickness, mass and moment of inertia.

\section{Introduction}

The global gravity data provide an important constraint on the lunar crustal thickness. The gravity inversion for a crustal thickness from topography and gravity data requires the assumption of the crust density, mantle density, and the reference radius of the crust-mantle boundary. The mantle relief derived in this way is highly dependent on the assumed density gap at the crust-mantle boundary.

Although the crustal densities of Zuber et al. (1994) and Neumann et al. (1996) were based on the best available values of nonmare lunar samples, and Wieczorek and Phillips (1998) derived a dual-layered crust model on the basis of several geophysical and petrological evidences, they modeled density contrast at the crust-mantle boundary without a detailed discussion.

The reference radii of the crust-mantle boundary used in Zuber et al. (1994) and Neumann et al. (1996) are constrained so that the crustal thickness at the Apollo 12 site is set to about $55 \mathrm{~km}$ (Nakamura et al., 1979), and that used in Wieczorek and Phillips (1998) is determined so that thickness at the Apollo 12 site is set to $60 \mathrm{~km}$ (Toksöz et al., 1974). The recent re-analyses of the Apollo seismic data, however, suggest a significantly thinner crust in a range of about 30-40 km around the Apollo 12 site (Khan and Mosegaard, 2002; Lognonné et al., 2003). Therefore the validity of the three parameters used in previous gravityinversion studies must be re-examined in a more careful

Copyright (c) The Society of Geomagnetism and Earth, Planetary and Space Sciences (SGEPSS); The Seismological Society of Japan; The Volcanological Society of Japan; The Geodetic Society of Japan; The Japanese Society for Planetary Sciences; TERRAPUB way.

In this study, by using a simple three-layered mass distribution model of the Moon that consists of a uniform crust, mantle, and core, we derive possible combinations of the three parameters, satisfying the mass and moment of inertia constraints of the Moon. We examine sets of the three parameters used in previous gravity-inversion studies, by using the method developed in this study.

\section{Methods}

The density distribution in the lunar interior is largely constrained by the measured total mass and the moment of inertia. In this study, we consider a simple three-layered model of the lunar interior (radially-symmetric), which consists of a uniform crust (the density of $\rho_{C r}$ ), mantle (the density of $\rho_{M}$ ), and core (the density of $\rho_{C o}$ ), and derive possible combinations of $\rho_{C r}, \rho_{M}$, and the radius of crust-mantle boundary $R_{M}$, satisfying the above constraints. In this model, the radius of the core-mantle boundaries are defined by $R_{C o}$, and the mean radius of the Moon $(R=1737.1$ $\mathrm{km}$; Smith et al., 1997) is used as the surface of the model. Gravitational constant $G$ times mass $M$ and the average moment inertia factor of the Moon are $G M=4902.8003 \pm$ $0.0012 \mathrm{~km}^{3} / \mathrm{s}^{2}$ and $I / M R^{2}=0.3931 \pm 0.0002$ respectively (Konopliv et al., 1998). Using gravitational constant recommended by 'CODATA-86' $(G=6.67259 \pm 0.00085$ $\mathrm{m}^{3} / \mathrm{kgs}^{2}$; Cohen and Taylor, 1987), the total mass of the Moon is estimated as $M=7.34767 \pm 0.0009410^{22} \mathrm{~kg}$. If $\rho_{C r}, \rho_{C o}$, and $R_{C o}$ are assumed, we can derive a $R_{M} v s . \rho_{M}$ relation by solving the two equations concerning $M$ and $I$ simultaneously.

Figure 1 shows resultant relations between the $R_{M}$ and 


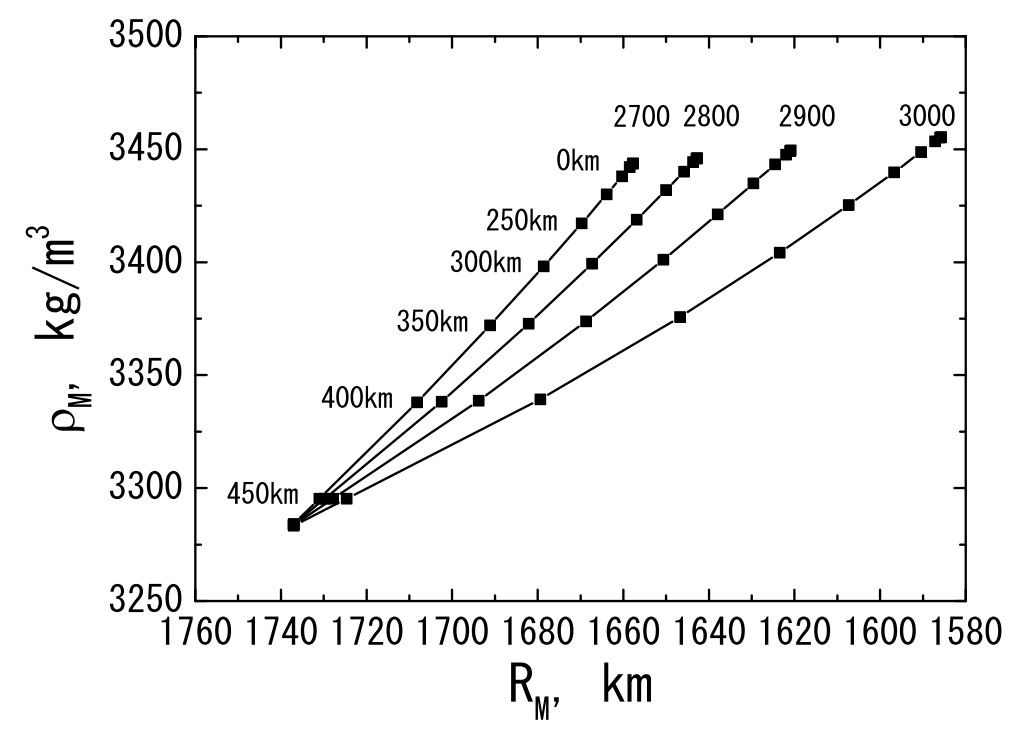

Fig. 1. The $R_{M}$ vs. $\rho_{M}$ relation constrained by the mass and moment of inertia for several assumed crustal densities. The assumed core sizes are also shown, where the core densities are assumed to be $6600 \mathrm{~kg} / \mathrm{m}^{3}$ for all the cases.

$\rho_{M}$ relation for various assumed crustal densities ranging from 2700 to $3000 \mathrm{~kg} / \mathrm{m}^{3}$ (the values of the crustal densities are shown in $\mathrm{kg} / \mathrm{m}^{3}$ above each line). The crustal density of $2700 \mathrm{~kg} / \mathrm{m}^{3}$ is below the normative densities of nonmare lunar samples (2800 to $3000 \mathrm{~kg} / \mathrm{m}^{3}$; Papike, 1998), but is possible if the effect of porosity within the crust is considered. We assume here the core density of $6600 \mathrm{~kg} / \mathrm{m}^{3}$, which corresponds to the density of the Earth's core at 1 atmospheric pressure (Anderson, 1989). The assumption of the core density is somewhat arbitrary. As is discussed in the next section, a resultant $R_{M} v s . \rho_{M}$ relation is found to significantly change, as $\rho_{C o}$ changes. In Fig. 1 , the assumed core sizes are shown along the lines. The plots along each line are shown at an interval of $50 \mathrm{~km}$ in core size beginning from $0 \mathrm{~km}$ at the uppermost of the line. We can see that the crustal density, mantle density, and the reference radius of the crust-mantle boundary are correlated each other so that these values should not be determined independently, which is an ignored point in the previous studies of the gravity inversion for the crustal thickness of the Moon.

\section{Discussions}

\subsection{Model uncertainties}

Although the relation between $R_{M}$ and $\rho_{M}$ is derived by using a simple three-layered mass distribution model, there could be uncertinties inherent with this simplified model. In this section, we examine model uncertainties, and estimate these effects on $R_{M} v s$. $\rho_{M}$ relation.

First, we estimate contributions of the topography and mantle relief along the crust-mantle boundary to the mass and moment of inertia. Because the crust lies at the outer most part of the Moon, the topographic contribution as well as the contribution of mantle relief with density gap along the crust-mantle boundary may not be negligible. The topographic contributions from the mean radius of the Moon are calculated by using the recent developed topography model of 90th-degree and order obtained by Clementine LIDAR (Nozette et al., 1994; Smith et al., 1997), and the results show that the contributions to the total mass amd the moment of inertia are less than $0.02 \%$ of $M$ and $0.03 \%$ of $I$ respectively.

The contributions of the mantle relief along the crustmantle boundary are also calculated, where the relief is derived so that the gravitational anomalies due to the relief with the density gap $\left(\rho_{M}-\rho_{C r}\right)$ satisfy the Bouguer anomalies, residual gravitational anomalies subtracting the gravitational attractions due to topography (the Bouguer correction) from observed gravitaional anomalies. In this calculation, the mantle relief is expanded in the spherical harmonic form. The degree $n-$ and order $m-$ normalized spherical harmonic coefficients of the mantle relief are written as;

$$
\left(\begin{array}{c}
H_{n m}^{C} \\
H_{n m}^{S}
\end{array}\right)=\left(\frac{R_{\mathrm{ref}}}{R_{M}}\right)^{n} \frac{(2 n+1) M}{4 \pi\left(\rho_{M}-\rho_{C r}\right) R_{M}^{2}}\left(\begin{array}{c}
C_{n m}^{\mathrm{BA}} \\
S_{n m}^{\mathrm{BA}}
\end{array}\right)
$$

where $R_{\text {ref }}$ is the reference radius assumed by the gravity model $\left(1738 \mathrm{~km}\right.$; Konopliv et al., 2001). $C_{n m}^{\mathrm{BA}}$ and $S_{n m}^{\mathrm{BA}}$ are the harmonic coefficients of the Bouguer anomalies. We assume here that the mantle relief is much smaller than the reference radius of the crust-mantle boundary. We use 165th-degree gravity model (LP165P; Konopliv et al., 2001) as the observed data, which is determined from the orbital tracking data of the Lunar Prospector spacecraft (Binder, 1998) as well as from previous missions (The Lunar Orbiter I-V, the Apollo 15 and 16 subsatellites, and the Clementine spacecraft). We expand the mantle relief in the spherical harmonics up to the degree and order of 25 , which is based on the fact that the power spectra of the Bouguer correction match the spectra of the observed gravitational anomalies within the data errors at a given degree higher than 25. In deriving the mantle relief, the gravitational attraction due to surface mare flows is neglected, because our present purpose is to estimate the effect of topography and realistic mantle relief on the $R_{M} v s . \rho_{M}$ relation satisfying the mass and moment of inertia constraints. The effect of the mare basalt flows does not change the result significantly concerning the relief contributions to the mass and 


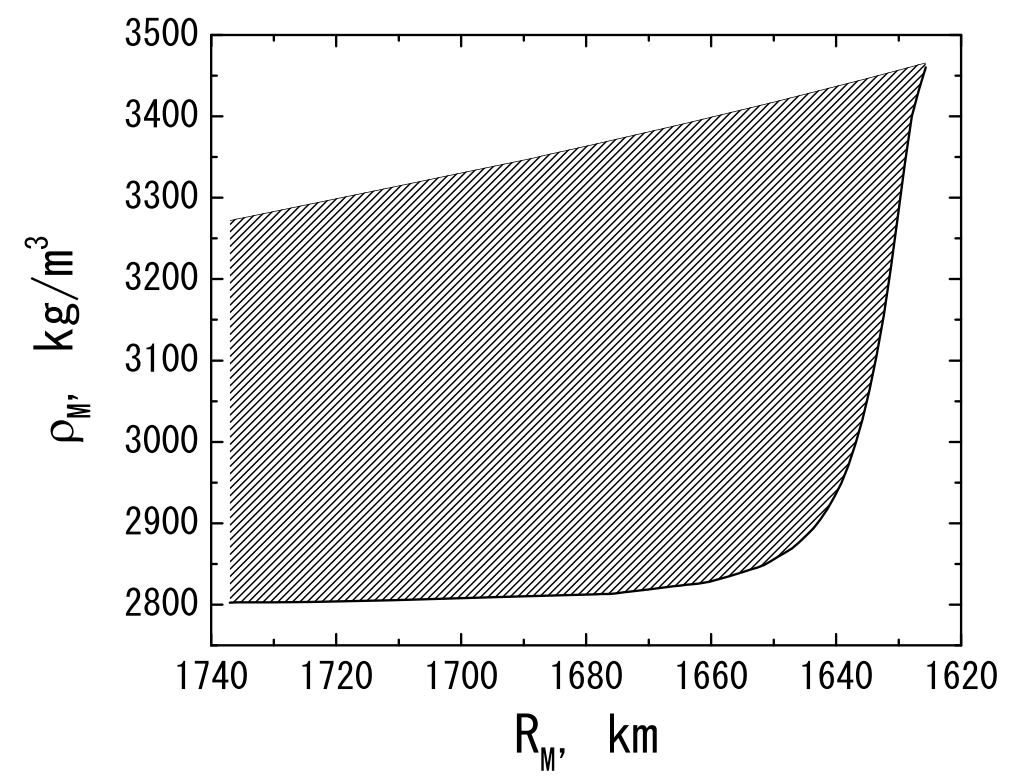

Fig. 2. The uncertainty of the mantle density as a function of $R_{M}$ (the upper and lower bound), where the crustal density is assumed to be $2800 \mathrm{~kg} / \mathrm{m}^{3}$.

moment of inertia. The result show that the mantle relief contributions are found to be about $0.1 \%$ of $M$ and about $0.2 \%$ of $I$ respectively.

The sum of the topographic and mantle relief contributions to the mass and moment of inertia are $0.15 \%$ of $M$ and $0.25 \%$ of $I$ respectively, which are larger than the uncertainties of the mass and moment of inertia by order (the uncertainties of $M$ and $I$ are $0.01 \%$ and $0.06 \%$ respectively). Therefore the mass and moment of inertia values, which are used in the simple three-layered model of the Moon (without any reliefs), must be corrected in this sense. We subtract the topographic and mantle relief contributions from the observed mass and moment of inertia, and use the residual mass and moment of inertia as constraints in the following discussion. These corrected values of $I$ and $M$ are presented by $M_{\text {cor }} \equiv M-M_{\text {rlf }}$ and $I_{\text {cor }} \equiv I-I_{\text {rlf }}$ respectively, where $M_{\text {rlf }}$ and $I_{\text {rlf }}$ are the topographic and mantle relief contributions to the mass and moment of inertia respectively.

Second, we derive upper and lower bound of the mantle density at a given $R_{M}$, using the uncertainties of the mass and moment of inertia, as well as changing the core density in a reasonable range. For the relations among $\rho_{C r}, \rho_{M}$, and $\rho_{C} O$, we assign the following condition on the basis of the idea that material is always denser with depth within the Moon;

$$
\rho_{C r} \leq \rho_{M} \leq \rho_{C o} \leq \rho_{\gamma F e}
$$

where $\rho_{\gamma F e}$ represents the maximum core density, and is assumed to be that of $\gamma$-Fe core $\left(8100 \mathrm{~kg} / \mathrm{m}^{3}\right)$. The density of $\gamma$-Fe is estimeted from the available experimental data (Kuskov, 1995). The upper bound of $\rho_{M}$ at a given $R_{M}$ is derived by using the maximum core density, $\rho_{\gamma F e}$, and the allowable maximum values of the mass and moment of inertia, $\left(M_{\text {cor }}+M_{\text {err }}\right)$ and $\left(I_{\text {cor }}+I_{\text {err }}\right)$, where $M_{\text {err }}$ and $I_{\text {err }}$ are the uncertainties of the observed mass and moment of inertia respectively. In the same way, the lower bound of $\rho_{M}$ at a given $R_{M}$ is derived by using the minimum core density, which is equivalent to the mantle density, and the allowable minimum values of the mass and moment of inertia, $\left(M_{\text {cor }}-M_{\text {err }}\right)$ and $\left(I_{\text {cor }}-I_{\text {err }}\right)$.

The results are shown in Fig. 2 for the case of $\rho_{C r}=2800$ $\mathrm{kg} / \mathrm{m}^{3}$. It should be noted that the $R_{M}$ vs. $\rho_{M}$ relation for $\rho_{C r}=2800 \mathrm{~kg} / \mathrm{m}^{3}$ of Fig. 1 is in the upper region of the allowable range shown in Fig. 2. This is because in Fig. 1, we does not correct the contribution of topography and mantle relief to the observed mass and moment of inertia. As mentioned previously, the topographic and mantle relief contribution are much larger than the uncertainties of the mass and moment of inertia. Therefore $\left(M_{\mathrm{cor}}+M_{\mathrm{err}}\right)$ and $\left(I_{\text {cor }}+I_{\text {err }}\right)$, which is used for deriving the upper bound of $\rho_{M}$ in Fig. 2, are always smaller than $I$ and $M$, which is used for deriving $R_{M}$ vs. $\rho_{M}$ relation of Fig. 1. As increasing the values of the total mass and moment of inertia used in the calculation increase the resultant value of $\rho_{M}$ at a given $R_{M}$, the $R_{M}$ vs. $\rho_{M}$ relation of Fig. 1 is beyond the allowable range shown in Fig. 2.

In deriving a lower bound of $\rho_{M}$ in Fig. 2, the core density of $3460 \mathrm{~kg} / \mathrm{m}^{3}$ is used. For the core density less than $3460 \mathrm{~kg} / \mathrm{m}^{3}$, the mass model does not satisfy the condition of Eq. (2). The different assumption of the crustal density is found to derive almost the same results for the $\rho_{M}$ difference between the cases for the core densities of 6600 and $8100 \mathrm{~kg} / \mathrm{m}^{3}$. As is not shown in this paper, the degree of the difference of $\rho_{M}$ between the model for the core density of $6600 \mathrm{~kg} / \mathrm{m}^{3}$ and that for the minimum core density, however, varies with the assumption of crustal density.

We also examine the effect of vertical density variation in the crust. Hikida and Mizutani (in preparation) derives a vertical density distribution model of the lunar crust, by using compressional and shear-wave velocities measurement of the Apollo rocks with pressure. Their model is based on the idea that crustal materials are porous near the surface due to the heavy bombardment of meteorite impacts on the surface and become gradually denser with depth due to the pore closures by lithostatic pressure as suggested by 
seismic velocity model (Toksöz, et al., 1974). According to their model, crustal material typical of the lunar highland $(5.5 \mathrm{wt} \% \mathrm{FeO}$ inclusion, the modal iron abundance of the lunar surface from the Lunar Prospector Gamma-Ray Spectrometer, Lawrence et al., 2002) increases its density with depth as;

$$
\begin{aligned}
\rho_{C r}(r)= & {\left[1.35074-4.34563\left(\frac{r}{R}\right)+4.66990\left(\frac{r}{R}\right)^{2}\right.} \\
& \left.-1.67264\left(\frac{r}{R}\right)^{3}\right]
\end{aligned}
$$

where $r$ is the radial distance from the gravitational center of the Moon, and the unit of the equation is $10^{6} \mathrm{~kg} / \mathrm{m}^{3}$. Using the Eq. (3), the resultant value of $\rho_{M}$ at a given $R_{M}$ is found to be at most $0.5 \%$ higher than that of the upper bound of Fig. 2. Although there are highland samples which has higher iron abundances than $5.5 \mathrm{wt} \%$, Hikida and Mizutani (in preparation) shows that the assumption of the higher abundances of iron in the crust, e.g., $10 \mathrm{wt} \%$, which is much lower proportion in the iron map of Lawrence et al. (2002) in comparison to that for $5.5 \mathrm{wt} \%$, does not change the resultant density variation in the crust so much. Note that the result for the vertical variation in the crust is not included in Fig. 2, nor is considered in the following section concerning the comparison with the previous gravity inversion studies, because the crustal densities in the previous studies are constant. Although Wieczorek and Phillips (1998) considers vertical density variation in the crustal, it is not gradually changed like the model presented here.

Although our intention is not to determine a detailed structure of the mantle, we discuss the uncertainties due to the simple assumption of uniform mantle, comparing with the lunar internal model of Kuskov et al. (2002). They derive a five-layer model of the Moon, including a uniform crust with $3000 \mathrm{~kg} / \mathrm{m}^{3}$ for the density and $60 \mathrm{~km}$ for the crustal thickness, three-layer mantle whose depths of the boundaries are based on the seismic analysis (Nakamura, 1983), and Fe-FeS-core, satisfying the mass and moment of inertia requirements. Using the Monte-Carlo method, they show numerous possible mass distribution models of the Moon and constrain the density range of each mantle layer, assuming that there is no inversion in the density profile within mantle (i.e., $\rho_{3} \geq \rho_{2} \geq \rho_{1}$, where $\rho_{1}, \rho_{2}$, and $\rho_{3}$ are the densities in the upper, middle, and lower mantle, respectively). In Kuskov et al. (2002), the density range of the mantle is also converted into the seismic velocities range from petrological models, which reveals that the density range for the upper mantle of Kuskov et al. (2002) is consistent with the seismic model for the upper mantle of Nakamura (1983). According to Kuskov et al. (2002), the allowable range for the upper-mantle density is between $3220 \mathrm{~kg} / \mathrm{m}^{3}$ and $3340 \mathrm{~kg} / \mathrm{m}^{3}$.

The upper mantle density is always lower than that in uniform mantle if the mantle is stratified in density and increases its density with depth. The maximum value of the upper-mantle density is the same as that derived in our model for the case of $\rho_{C r}=3000 \mathrm{~kg} / \mathrm{m}^{3}$ and $R_{M}=1677$ $\mathrm{km}$ (see Fig. 1). We conclude that the density of uniform mantle in our study has the uncertainty of (3220-
$3340) \times 100 / 3340 \sim-3.5 \%$, although the uncertainty depends weakly on the crustal density and much on the crustal thickness. Because the density range of the upper mantle of the three-layered mantle model is not estimated for all the range of $\rho_{C r}$ and $R_{M}$ in Kuskov et al. (2002), we does not include it in Fig. 2 (it is considered in the following section concerning the comparison with the previous gravity inversion studies.).

As mentioned above, Kuskov et al. (2002) does not consider any density inversion and/or decrease in the mantle with depth (i.e., the case which does not satisfy the condition of $\rho_{3} \geq \rho_{2} \geq \rho_{1}$ ). We have to discuss this possibility, because the pressure is low in the lunar mantle, and the effect of temperature increase dominates on the seismic profiles, as pointed out by Goins (1981). Although the mantle density model of Kuskov et al. (2002) is derived on the assumption of $\rho_{3} \geq \rho_{2} \geq \rho_{1}$, the density ranges of the middle and lower mantle of Kuskov et al. (2002) are useful for interpreting the effect of the density inverse in the mantle on the upper mantle density as an example.

A set of the middle and lower mantle densities of 3290 and $3340 \mathrm{~kg} / \mathrm{m}^{2}$, whose values are the lower bound of the middle and lower mantles of Kuskov et al. (2002), respectively, is consistent with the seismic model of Nakamura (1983). Note that the associated seismic velocities of the lower mantle with the density of $3340 \mathrm{~kg} / \mathrm{m}^{3}$ is smaller than those of Nakamura (1983), but has a good agreement if the fact that the seismic velocities of the lower mantle of Nakamura (1983) is overestimated by about $2 \%$ is considered (Kuskov et al., 2002). Assuming that the mantle is stratified by the three layers as assumed in Kuskov et al. (2002), and that the middle and lower mantle densities are 3290 and $3340 \mathrm{~kg} / \mathrm{m}^{2}$, respectively, the usage of the best values of the mass and moment of inertia, $M$ and $I$, as well as the maximum core density of $8100 \mathrm{~kg} / \mathrm{m}^{3}$ leads to the resultant upper mantle density of about $3370 \mathrm{~kg} / \mathrm{m}^{3}$ for the case of $\rho_{C r}=3000 \mathrm{~kg} / \mathrm{m}^{3}$ and $R_{M}=1677 \mathrm{~km}$. This value is larger than the result of our study (uniform mantle) by $(3370-3340) \times 100 / 3340 \sim+0.9 \%$.

From the estimation above, we can see that there is a possibility that the upper mantle density is larger than that in uniform mantle (this study) if the mantle is stratified in density and has density inversion and/or decrease with depth within the deeper mantle. However, because the example presented here is not unique (note again that the density ranges of the middle and lower mantles of Kuskov et al. (2002) are derived from the condition of $\rho_{3} \geq \rho_{2} \geq \rho_{1}$ ), we does not definitely determine the uncertainty due to the stratified mantle with density inversion within the deeper mantle. Therefore we conclude here that the upper bound of mantle density is potentially larger than that discussed in this study by $0.9 \%$, more or less. Although we does not include this uncertainty in Fig. 2, it is considered in the following section concerning the comparison with the previous gravity inversion studies. Clearly, the deeper mantle structure should be constrained from petrological and seismic analyses in the future work.

\subsection{Comparison with the previous studies}

In this section, we examine the validity of the three parameters $\left(R_{M}, \rho_{C r}, \rho_{M}\right)$ used in previous crustal thickness 
models from gravity inversions, by using the method developed in this study. The crustal thickness model of Zuber et al. (1994) is a preliminary one of Neumann et al. (1996), and the three parameters used in the model of Zuber et al. (1994) are the same as those of Neumann et al. (1996). Therefore, we examine only the two models of Neumann et al. (1996) and Wieczorek and Phillips (1998).

A uniform crust and mantle (the densities of $2800 \mathrm{~kg} / \mathrm{m}^{3}$ and $3300 \mathrm{~kg} / \mathrm{m}^{3}$ respectively) are assumed in the crustal model of Neumann et al. (1996). Their model is derived by anchoring the crustal thickness of $55 \mathrm{~km}$ near the Apollo 12 and 14 landing sites, where the reference radius of the mantle is $61 \mathrm{~km}$ below a nominal equatorial radius of 1738 $\mathrm{km}$, i.e., $R_{M}=1677 \mathrm{~km}$. For the radially-symmetric model developed in this study, the mantle density at $R_{M}=1677$ $\mathrm{km}$ is found to be $3380 \mathrm{~kg} / \mathrm{m}^{3}$, assuming the densities of the crust and core of 2800 and $6600 \mathrm{~kg} / \mathrm{m}^{3}$ respectively (Fig. 1), The value of the mantle density used in Neumann et al. (1996), is smaller than the value expected in Fig. 1, which suggests their model is inconsistent with the mass and moment of inertia constraints. However, the allowable range of $\rho_{M}$ at $R_{M}=1677 \mathrm{~km}$ in Fig. 2 is 2810 to 3370 $\mathrm{kg} / \mathrm{m}^{3}$ (the upper bound of $\rho_{M}$ is $10 \mathrm{~kg} / \mathrm{m}^{3}$ smaller than that derived without any uncertainties, because the correction for the topographic and mantle relief contribution to the mass and moment of inertia decreases the resultant mantle density, as discussed in the previous section). In addition to this, integrating the possibility that the mantle is vertically varied in density $\left(-3.5 \%\right.$ of $\rho_{M}$ if the mantle increases its density with depth, and $+0.9 \%$ of $\rho_{M}$ if the mantle has inversion in the density profile, the latter of which is a nominal value as discussed in the previous section), the range is widened to be 2710 to $3400 \mathrm{~kg} / \mathrm{m}^{3}$. As in this study, we assume that the mantle density is always denser than the crustal density, a realistic range of $\rho_{M}$ is then 2800 to 3400 $\mathrm{kg} / \mathrm{m}^{3}$ for $\rho_{C r}=2800 \mathrm{~kg} / \mathrm{m}^{3}$. The mantle density used in Neumann et al. (1996) lies within this range, indicating their model is consistent with the mass and moment of inertia, if the uncertainty is considered.

The crustal thickness model of Wieczorek and Phillips (1998) considers the upper anorthositic crust $\left(2800 \mathrm{~kg} / \mathrm{m}^{3}\right)$ and the lower crust of more mafic materials $\left(3100 \mathrm{~kg} / \mathrm{m}^{3}\right)$ on the basis of several geophysical and petrological evidences. Their model had an average $31 \mathrm{~km}$-thick upper crust and a $29 \mathrm{~km}$-thick lower crust (the total crustal thickness of $60 \mathrm{~km}$ ) in order to match the crustal thickness of 60 $\mathrm{km}$ at the Apollo 12 and 14 sites, assuming $\rho_{M}=3400$ $\mathrm{kg} / \mathrm{m}^{3}$. By using our three-layered model of the Moon, where the model is properly modified so that the crust is divided into two radially-symmetric layers (i.e., $31 \mathrm{~km}$-thick upper crust with the density of $2800 \mathrm{~kg} / \mathrm{m}^{3}$ and $29 \mathrm{~km}$-thick lower crust with the density of $3100 \mathrm{~kg} / \mathrm{m}^{3}$ ), and assuming the core density of $6600 \mathrm{~kg} / \mathrm{m}^{3}$, the resultant mantle density at $R_{M}=1677 \mathrm{~km}$ is found to be $3350 \mathrm{~kg} / \mathrm{m}^{3}$.

Considering the uncertainties discussed in the previous section (i.e., the errors of the mass and moment of inertia, the uncertainties of the core density, the effect of topography and mantle relief, and the effect of vertical density variation in the mantle), and constraining the mantle density to be larger than that of the crust (here the lower crustal den- sity, $3100 \mathrm{~kg} / \mathrm{m}^{3}$, is used), the allowable range of the mantle density is found to be 3100 to $3370 \mathrm{~kg} / \mathrm{m}^{3}$. This shows that the mantle density used in Wieczorek and Phillips (1998) does not lie within the allowable range, indicating that their model is inconsistent with the mass and moment of inertia constraints. According to Wieczorek (2003), the overweight of about $50 \mathrm{~kg} / \mathrm{m}^{3}$ of the mantle density leads to the increase of about $10 \mathrm{~km}$ of the crustal thickness around the Apollo 12 site, although the overweight can be compensated by adjusting both the densities of crust and mantle by the same quantities for the density gap to be the same (e.g., the crust and mantle densities of 3100 and $3400 \mathrm{~kg} / \mathrm{m}^{3}$ can be changed to those of 3050 and $3350 \mathrm{~kg} / \mathrm{m}^{3}$, in this case), which will not alter the major findings of their work so much.

\section{Conclusions}

For possible densities of the crust and core, the relation between the mantle density and the reference radius of the crust-mantle boundary is derived as a function of the size of the core. Our results from the mass and moment of inertia constraints of the Moon show that the three parameters of the crust density, the mantle density, and the reference radius of the crust-mantle boundary, which are needed for constructing the crustal structure from gravity inversions, are closely related to each other, and should not be determined independently. The results also show that the set of the parameters used in Neumann et al. (1996) can be consistent with mass and moment of inertia constraints, while that used in Wieczorek and Phillips (1998) does not satisfy the constraints.

Acknowledgments. We thank Gregory A. Neumann, Noriyuki Namiki, and Mark Wieczorek for reviews which greatly improved the manuscript.

\section{References}

Anderson, D. L., Theory of the Earth, 366 pp., Blackwell, Boston, MA, 1989.

Binder, A. B., Lunar Prospector: Overview, Science, 281, 1475-1476, 1998.

Cohen, E. R. and B. N. Taylor, The 1986 adjustment of the fundamental physical constants, Rev. Mod. Phys., 59, 1121-1148, 1987.

Goins, N. R., A. M. Dainty, and M. N. Toksöz, Lunar seismology, the internal structure of the Moon, J. Geophys. Res., 86, 5061-5074, 1981.

Khan, A. and K. Mosegaard, An inquiry into the lunar interior: A nonlinear inversion of the Apollo lunar seismic data, J. Geophys. Res., 107(E6), 3-1-3-23, 2002.

Konopliv, A. S., A. B. Binder, L. L. Hood, A. B. Kucinskas, W. L. Sjogren, and J. G. Williams, Improved gravity field of the Moon from Lunar Prospector, Science, 281, 1476-1480, 1998.

Konopliv, A. S., S. W. Asmar, E. Carranza, W. L. Sjogren, and D. N. Yuan, Recent gravity models as a result of the Lunar Prospector mission, Icarus, 150, 1-18, 2001.

Kuskov, O. L., Composition and structure of the upper mantle of the Moon, Geokhimiya, 12, 1683-1698, 1995.

Kuskov, O. L., V. A. Kronrod, and L. L. Hood, Geochemical constraints on the seismic properties of the lunar mantle, Phys. Earth Planet. Inter., 134, 175-189, 2002.

Lawrence, D. J., W. C. Feldman, R. C. Elphic, R. C. Little, T. H. Prettyman, S. Maurice, P. G. Lucey, and A. B. Binder, Iron abundances on the lunar surface as measured by the Lunar Prospector gamma-ray and nutron spectrometers, J. Geophys. Res., 107, 13-1-13-26, 2002.

Lognonné, P., J. Gagnepain-Beyneix, and H. Chenet, A new seismic model of the Moon: implication for structure, thermal evolution and formation of the Moon, Earth Planet. Sci. Lett., 211, 27-44, 2003. 
Nakamura, Y., Seismic velocity structure of the lunar mantle, J. Geophys. Res., 88, 677-686, 1983.

Nakamura, Y., G. V. Latham, H. J. Dorman, A.-B. K. Ibrahim, J. Koyama, and P. Horvath, Shallow moonquakes-Depth, distribution and implications as to the present state of the lunar interior, Proc. Lunar Planet. Sci. Conf., 10th, 2299-2309, 1979.

Neumann, G. A., M. T. Zuber, D. E. Smith, and F. G. Lemoine, The lunar crust: global structure and signature of major basins, J. Geophys. Res., 101, 16841-16843, 1996.

Nozette et al., The Clementine mission to the Moon: Scientific overview, Science, 266, 1835-1839, 1994.

Papike, J. J., Planetary Materials. Reviews in Mineralogy Vol. 36, 1014 pp., Mineralogical Society of America, Washington, 1998.

Smith, D. E., M. T. Zuber, G. A. Neumann, and F. G. Lemoine, Topography of the Moon from the Clementine lidar, J. Geophys. Res., 102, 1519-
$1611,1997$.

Toksöz, M. N., A. M. Dainty, S. C. Solomon, and K. Anderson, Structure of the Moon, Rev. Geophys. Space Phys., 12, 539-567, 1974.

Wieczorek, M. A., The thickness of the lunar crust: How low can we go?, Pros. Lunar. Planet. Sci., XXXIV, 1330, 2003.

Wieczorek, M. A. and R. J. Phillips, Potential anomalies on a sphere: Applications to the thickness of the lunar crust, J. Geophys. Res., 103, 1715-1724, 1998.

Zuber, M. T., D. E. Smith, F. G. Lemoine, and G. A. Neumann, The shape and internal structure of the Moon from the Clementine mission, Science, 266, 1839-1843, 1994.

H. Hikida (e-mail: hajime@ipgp.jussieu.fr) and H. Mizutani 